\title{
Case studies in numerical simulation of crack trajectories in brittle materials
}

\author{
H. Jasarevic \\ Faculty of Civil Engineering, University of Sarajevo, Sarajevo (Bosnia and Herzegovina) \\ haris.jasarevi@g.gnsa.ba \\ S. Gagula \\ Faculty of Engineering and Natural Sciences, International University of Sarajevo, Sarajevo (Bosnia and Herzegovina) \\ sadina@ius.edu.ba
}

\begin{abstract}
Statistical Fracture Mechanics, formalism of few natural ideas is applied to simulation of crack trajectories in brittle material. The "diffusion approximation" of the crack diffusion model represents crack trajectories as a one-dimensional Wiener process with advantage of well-developed mathematical formalism and simplicity of creating computer generated realizations (fractal dimension $d=1.5$ ). However, the most of reported $d$ values are in the range 1.1-1.3. As a result, fractional integration of Wiener processes is applied for lowering $d$ and to generate computer simulated trajectories. Case studies on numerical simulation of experimentally observed crack trajectories in sandstone (discs tested in indirect tensile strength test) and concrete (compact tension specimens tested in the quasi-static splitting tensile test) are presented here.
\end{abstract}

KEYwORDS. Statistical Fracture Mechanics; Brittle materials; Crack trajectories; Fractal dimension; Sandstone; Concrete.

\section{INTRODUCTION}

\footnotetext{
pproach to describe the physics of fracture for cases when failure of single element does not equal to failure of whole body was proposed first by Chudnovsky [1]. It evolved afterwards in crack diffusion model [2] and Statistical Fracture Mechanics (SFM) [3, 4], which both describe crack propagation in brittle materials. SFM formalizes following natural ideas [4]:

i) crack advance consists of a sequence local fail ures in front of a crack tip,

ii) the local failures are random events due to fluctuations of local strength of the material,

iii) the crack trajectory is random, i.e. crack can follow any path from a set $\Omega$ of all admissible crack paths.

For each of those paths conditional probability of failure along that path exists. The probability of crack advancing from point $\mathrm{A}$ to point $\mathrm{B}$ is an average of those conditional probabilities over all admissible crack paths leading from $\mathrm{A}$ to $\mathrm{B}$.
}

\section{SIMULATION PROCEDURE}

7 he "diffusion approximation" of the crack diffusion model (see Chudnovsky and Gorelik, 1994), represents crack trajectories as a one-dimensional Wiener process (Brownian motion) $w(t)$. This approach has advantage due to well-developed mathematical formalism and simplicity of creating computer generated realizations with known fractal dimension $d=1.5$. However, often it cannot be directly applied to simulate actually observed fracture profiles, 
since the most of reported $d$ values are in the range 1.1-1.3 [5]. As a result, it is necessary to modify $w(t)$ to lower its $d$. The simulation procedure by applying the operation of fractional integration to realization of $w(x)$ in order to decrease $d$ to desired value is described by Kunin and Gorelik [5] in detail.

The realization $w(x)$ with $d=1.5$ is first generated as integral of a white noise $e(x)$ written in discrete form as:

$$
w(x)=D \sum_{(i)} e\left(\xi_{i}\right) \Delta_{i} \xi
$$

where $e_{i}$ is Gaussian random variable with zero mean and unit variance and $D$ is crack diffusion coefficient determined from experimental data. $D$ is statistical parameter reflecting the tendency of crack trajectories to deviate from central axis [5].

Once the realization is generated, it is necessary to modify fractal dimension by applying the fractional integration operation to it as follows:

$$
w_{\lambda}(x)=\frac{1}{\Gamma(\lambda)} \int_{0}^{x} w(\xi)(x-\xi)^{\lambda-1} d \xi
$$

Where $\Gamma(\ldots)$ is the Gamma-function, and fractal dimension of $w_{\lambda}(x)$ is $d=1.5-\lambda$. A numerical implementation of above described procedure has been coded into the software MatLab ${ }^{\circledR}$ for case study simulations presented later in this text.

The algorithm requires 3 input parameters, namely $d, D$ and $n$ (number of trajectories to be generated). Each realization of $w(x)$ with $d=1.5$ incorporating parameter $D$ is generated with 1000 time steps (intervals) and fractional integration operation is performed to create final realization $w_{\lambda}(x)$ with $d=1.5-\lambda$. The process is repeated in DO loop till $n$ trajectories are created. Due to the above described formalism simplicity and computational performance of modern computers, this whole process is completed in the matter of couple of seconds. However, the major issue is obtaining input parameters $(d$, $D$ ) for simulation from experiments (sufficient number of repeated experimental tests is needed). It should be noted that both parameters are strongly scale dependent. Issa at al [6] stated that groups of identically sized concrete specimens prepared with different aggregate sizes have different values of $D$ (one with smaller size aggregate have smaller value of $D$ ). In addition, larger sized specimens with identical mix have lower values of $d$. Also, Zavarise at al [7] pointed that statistical parameters used in stochastic contact models are scale dependent (being function of profilometer resolution).

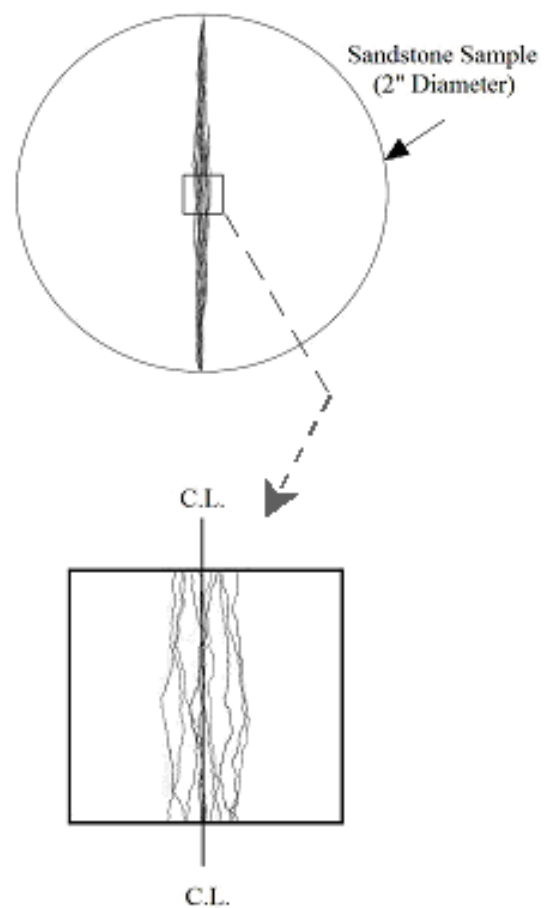

Figure 1: Experimentally observed crack trajectories in sandstone discs.

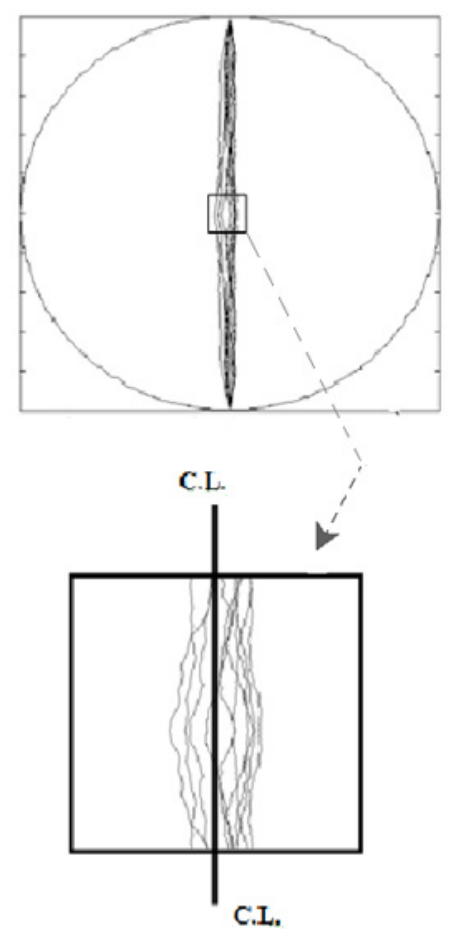

Figure 2: Simulated sandstone crack trajectories with $d=1.2$ and $D=0.01$ 


\section{CRACK TRAJECTORIES SIMULATION CASE STUDIES}

\section{Sandstone}

7 he test results on macroscopically identical sandstone discs tested in indirect tensile strength test (ASTM [8] and ISRM [9]) were reported by Jasarevic at al [10,11]. $50.8 \mathrm{~mm}$ diameter and $25.4 \mathrm{~mm}$ thickness ( $\mathrm{t} / \mathrm{D}=0.5$ ) discs cut from a block of Torry Buff sandstone are used for the tests. The Torry Buff material tested is a very fine-grained porous, consolidated sandstone, porosity $\sim 19 \%$, permeability $\sim 3$-millidarcy, Young's modulus $10.5 \mathrm{MPa}$ (measured on three 44.5-mm diameter, 92.4-mm length cylinders) and dry unconfined compressive strength $\sim 40 \mathrm{MPa}$. Total of ten discs were tested and observed crack trajectories are shown in Fig. 1 (Jasarevic, 2009a). Results clearly show that crack trajectories are random (i.e. no two coincide) satisfying SFM formalism described before. Result of computer simulated crack trajectories for fractal dimension $d=1.2$ and $D=0.01$ (see Jasarevic [10] for calculation details) is shown in Fig. 2 for comparison.

\section{Concrete}

The test results for study of size effects in concrete fracture on multiple sizes of macroscopically identical compact tension specimens tested in the quasi-static splitting tensile test (ramp test) were reported by Issa at al $[6,12]$. The specimens were positioned so that the notch was at the top and the actuator applied the load downward through a wedge at an angle of $8.7^{\circ}$. A wedge applied the load on the rollers that passed through rectangular cylinders. The rectangular cylinders had the same dimensions as the rectangular part of the notch. The downward force applied through a wedge was translated into a splitting tensile force through the rollers. The test was conducted in the displacement control mode at a rate of loading of $0.125 \mathrm{~mm} / \mathrm{min}$. Some of conclusions of this work are that concrete fracture surfaces with the larger aggregate sizes appear to have a higher roughness than those with smaller aggregate sizes. The crack path is less tortuous for geometrically identical specimens with smaller size aggregates. Similarly, the crack path deviates from the centerline of the specimen to a less degree for the smaller size aggregates than that for the larger ones. Crack trajectories for 6 macroscopically identical specimens with maximum aggregate size of $3 / 4$ in. $(19 \mathrm{~mm})$ are shown in Fig. 3. Same as for sandstone, experimentally observed crack path appears to be random, i.e., no two macroscopically identical specimens exhibit the same fracture path. Result of computer simulated crack trajectories for fractal dimension $d=1.1$ and $D=0.05$ (see Issa at al [12] and Hammad and Issa [13] for calculation details) is shown in Fig. 4 for comparison.

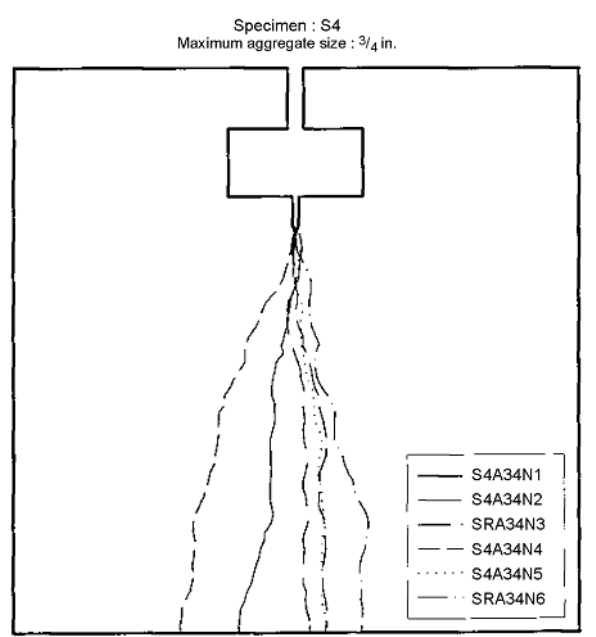

Figure 3: Experimentally observed crack trajectories in concrete specimens.

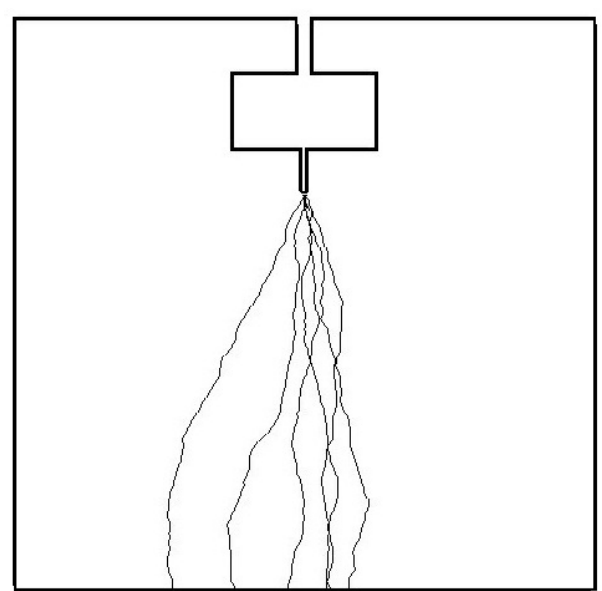

Figure 4: Simulated concrete crack trajectories for $d=1.1$ and $D=0.05$.

\section{CONCLUSION}

S andstone and concrete as brittle materials satisfy the SFM formalism in which no two fracture paths for macroscopically identical specimens coincide. The "diffusion approximation" of the crack diffusion model cannot be applied to crack trajectories experimentally observed in sandstone and concrete, since their fractal dimension is less then 1.5. Instead fractional integration of Wiener processes together with parameters extracted from experiments was 
used to simulate fracture profiles. Computer realizations of crack trajectories for two case studies presented here seem to be in good agreement with experimentally observed ones. Procedure for simulation is relatively simple and straightforward once the input parameters (fractal dimension $\mathrm{d}$ and diffusion coefficient $\mathrm{D}$ ) are known. However, sufficient number of repeated experimental tests is needed to extract these parameters.

\section{REFERENCES}

[1] A. Chudnovsky, In: Studies on Elasticity and Plasticity, ed. L. Kachanov, Leningrad, Leningrad University Press (1973) 3.

[2] A. Chudnovsky, B. Kunin, J. Appl. Phys., 62 (1987) 4124.

[3] A. Chudnovsky, B. Kunin, In: Microscopic Simulation of Complex Hydrodynamic Phenomena, eds. M. Mareschal and B.L. Holian, Plenum Press, New York (1992) 345.

[4] A. Chudnovsky, M. Gorelik, In: Probabilities and Materials, ed. D. Breysse, Netherlands, Kluwer Academic Publishers (1994) 321.

[5] B. Kunin, M. Gorelik, J. Appl. Phys. 70, (1991) 7651.

[6] M. A. Issa, M. A. Issa, H. Abdalla, M. S. Islam, A. Chudnovsky, Int. J. of Fract., 102 (2000) 25.

[7] G. Zavarise, M. Borri-Brunetto, M. Paggi, Wear, 262 (2007) 42.

[8] ASTM. Annual Book of Standards: Standard test method for splitting tensile strength of intact rock core specimens (designation D 4645-87). American Society for Testing and Materials, 4 (1989) 851.

[9] ISRM, Int. J. Rock Mech. Min, Sci. \& Geomech. Abstr., 15 (1978) 99.

[10] H. Jasarevic,. Observation, Characterization and Modeling of Fracture Initiation Phenomena, Ph.D thesis, Dept. of Engineering, Univ, Ill. at Chicago (2009).

[11] H. Jasarevic, A. Chudnovsky, J. W. Dudley, G. K. Wong, Int. J. Fract. , 158 (2009) 73.

[12] M. A. Issa, M. A. Issa, H. Abdalla, M. S. Islam, A.Chudnovsky, Int. J. of Fract., 102 (2000) 1.

[13] A. M. Hammad, M. A. Issa, Adv. Cem. Based Mater. 1 (1994) 169. 